\title{
Performance aspects of linear multiuser receivers
}

\author{
Brett Ninness and Steven R. Weller \\ Department of Electrical and Computer Engineering \\ University of Newcastle, Australia
}

\begin{abstract}
Recent analysis of linear multiuser receivers for DS-CDMA signals has considered signal-to-interference (SIR) performance in large systems with each user employing random spreading sequences. Unfortunately, it is only in the special case of the received powers of all users being equal that a resulting integral equation for SIR has an explicit solution with demonstrable approximation accuracy for finite-sized systems. This paper presents an alternative approach to calculating the SIR performance paper presents an alternative approach to calculating the SIR performance
of MMSE receivers which relies primarily on the law of large numbers, and provides an estimate for SIR that is valid for arbitrary distributions of received interferer powers.
\end{abstract}

\section{INTRODUCTION}

The problem considered here is that of minimum meansquare error (MMSE) detection of direct-sequence codedivision multiple access (DS-CDMA) signals, with a focus on providing a simple approximation of the interference experienced by a given user due to other users and background noise. The nature of study pursued here is inspired by recent independent works [1] and [2], and in particular the latter where attention is targeted on quantifying the signal-to-interference ratio (SIR) in such a manner that the contributions of the various effects of processing gain, received powers of interferers and background noise are clearly exposed.

There are two key tools employed in [2], [1]. The first is to model the spreading sequences of individual users as realizations of independent and identically distributed (i.i.d.) stochastic processes having zero mean and unit variance; see also [3]. The second is to recognize that the SIR, being a quadratic form of a random matrix, depends explicitly on the eigenvalue distribution of that random matrix. Recent results from the mathematical statistics literature [4] characterizing this distribution may then be employed to provide expressions of engineering relevance.

This approach leads to a key result [2, Theorem 3.1] that provides an approximation for the SIR under the assumption that both the number of users and the length of the spreading sequences are large, but that their ratio is fixed. Unfortunately, this expression is only available in an implicit form which requires the solution of a certain integral equation. As a consequence, in [2] a closed-form SIR approximation is only available for the special case when the received powers of the interferers are equal.

Furthermore, the methods used to derive the results in [2] involve relatively sophisticated machinery, including Stieltjes transforms and distributions which must be understood in a measure-theoretic sense. These may prove to be a limiting factor in the penetration of the results and the understanding of their genesis.

By way of contrast, this paper illustrates how SIR approximations for MMSE receivers may be derived using only very simple ideas, namely the Matrix Inversion Lemma and the Law of Large Numbers. The resulting closed-form expression for the asymptotic SIR is obtained for arbitrary distributions of received interferer powers.

\section{SIGNAL-TO-INTERFERENCE PERFORMANCE OF MMSE RECEIVERS}

Of interest is the following chip-sampled discrete-time model for a symbol-synchronous DS-CDMA system

$$
Y=\sum_{i=1}^{K} x_{i} S_{i}+W
$$

where $x_{i} \in \mathbf{R}$ is the symbol transmitted by the $i$ 'th user who possesses spreading sequence $S_{i} \in \mathbf{R}^{N}$. The length of the signature sequence $S_{i}$ is thus $N$, which is also termed the 'processing gain', and hence via (1) the received vector signal $Y \in \mathbf{R}^{N}$ consists of the linear superposition of the signals sent by all $K$ users together with the additive vector noise $W \in \mathbf{R}^{N}$. Here, as in [2], this noise will be modeled as white and Gaussian so that $W \sim \mathcal{N}\left(0, \sigma^{2} I\right)$.

Furthermore, the symbols $\left\{x_{i}\right\}$ will also be modeled as random variables, independent of one another, and such that the mean and variance satisfy

$$
\mathbf{E}\left\{x_{i}\right\}=0, \quad \mathbf{E}\left\{x_{i}^{2}\right\}=p_{i}
$$

so that $p_{i}$ is the received power of user number $i$.

For the purposes of demodulating the signal sent by user $i=$ 1 , it is then useful to think of the received signal as

$$
Y=x_{1} S_{1}+Z
$$

where now $Z \in \mathbf{R}^{N}$ represents a composite disturbance to the reception of the signal from user 1 , and consists of the received signals of all other users together with the background noise:

$$
Z \triangleq \sum_{i=2}^{K} x_{i} S_{i}+W
$$

Therefore, the variance of $Z$ conditional upon the receiver knowing the signature sequences $\left\{s_{2}, \cdots, s_{K}\right\}$ is

$$
P_{Z} \triangleq \mathbf{E}\left\{Z Z^{T} \mid S\right\}=S D S^{T}+\sigma^{2} I
$$

where

$$
S \triangleq\left[S_{2}, S_{3}, \cdots, S_{K}\right], \quad D=\left[\begin{array}{cccc}
p_{2} & 0 & \cdots & 0 \\
0 & p_{3} & & \\
\vdots & & \ddots & \\
0 & \cdots & \cdots & p_{K}
\end{array}\right]
$$

In order to form the demodulated estimate $\widehat{x}_{1}$ of the transmitted symbol $x_{1}$ the class of linear receivers of the form

$$
\widehat{x}_{1}=C^{T} Y, \quad C \in \mathbf{R}^{N}
$$

is of interest. As established in [5], the choice

$$
C=\left(p_{1} S_{1} S_{1}^{T}+P_{Z}\right)^{-1} p_{1} S_{1}=\frac{p_{1} P_{Z}^{-1} S_{1}}{1+p_{1} S_{1}^{T} P_{Z}^{-1} S_{1}}
$$


provides the optimal mean square error solution that minimizes both $\mathbf{E}\left\{\left(x_{1}-C^{T} Y\right)^{2} \mid S\right\}$ and

$$
\beta_{N} \triangleq \frac{p_{1} \mathrm{E}\left\{\left(C^{T} S_{1}\right)^{2} \mid S\right\}}{C^{T} P_{Z} C}
$$

over the class of all linear receivers. The quantity $\beta_{N}$ is the signal-to-interference ratio (SIR) for the reception of a particular user (in this case user number 1), and is a useful figure of merit in considering the performance of DS-CDMA systems [5], [2].

Substituting (4) into (3) implies that the optimal SIR for the class of linear receivers is

$$
\beta_{N}=p_{1} S_{1}^{T} P_{Z}^{-1} S_{1}=p_{1} S_{1}^{T}\left(S D S^{T}+\sigma^{2} I\right)^{-1} S_{1} .
$$

It is of interest to study this expression in order to gain insight into how the optimal SIR is affected by such factors as the distribution of received powers (according to the diagonal entries of $D$ ), and the ratio $\alpha=K / N$ of number of users $K$ to processing gain (spreading sequence length) $N$.

\section{PREVIOUS WORK}

Previous work has tackled the question of gaining insight into (5). In particular the recent studies [2], [1] have recognized that (5) is expressible as

$$
\beta_{N}=p_{1} \sum_{k=1}^{N} \frac{\left(u_{k}\right)^{2}}{\lambda_{k}+\sigma^{2}}
$$

where the numbers $\left\{\lambda_{k}\right\}$ are the eigenvalues of $S D S^{T}$ and the numbers $u_{k}$ are the projections of the elements of $S_{1}$ onto the unit length eigenvectors of $S D S^{T}$ (associated with the eigenvalues $\left\{\lambda_{k}\right\}$ in turn). If $g\left(\lambda_{k}\right)$ is then the number of eigenvalues in a region $\Delta_{k}$ centred on $\lambda_{k}$, then [2] argues that in some sense $u_{k}^{2} \approx g\left(\lambda_{k}\right) / \Delta_{k}$ and hence in the limit as $N \rightarrow \infty$ and as the regions $\Delta_{k}$ shrink,

$$
\lim _{N \rightarrow \infty} \beta_{N}=\beta=p_{1} \int_{0}^{\infty} \frac{g(\lambda)}{\lambda+\sigma^{2}} \mathrm{~d} \lambda .
$$

Obtaining further clarification then pivots on quantifying the eigenvalue density $g(\lambda)$. The key contribution in [2], [1] (see also [5]), is to model the spreading sequences $\left\{S_{k}\right\}$ as

$$
S_{k}=\frac{1}{\sqrt{N}}\left[V_{k}(1), \cdots, V_{k}(N)\right]
$$

where the elements $\left\{V_{k}(j)\right\}$ are identically distributed random variables that are all independent for different $k$ or $j$, and such that $E\left\{V_{k}(j)^{2}\right\}=1$ for all $k$ and $j$. For technical reasons it is also assumed that $E\left\{\left|V_{k}(j)\right|^{4}\right\}=\kappa<\infty$.

The results of [4] may then be applied to characterize $g(\lambda)$; specifically, $g$ is such that the following integral equation holds for all $z \in \mathbf{C}, \operatorname{Re}(z)>0$ :

$$
m(z)=\left(\alpha \int_{0}^{\infty} \frac{\tau f(\tau)}{1+\tau m(z)} \mathrm{d} \tau-z\right)^{-1}
$$

Here $\alpha=K / N, f(\tau)$ is the limiting density (i.e. limiting shape of the histogram) of the received powers making up the diagonal entries of $D$, and

$$
m(z)=\int_{0}^{\infty} \frac{g(\lambda)}{\lambda-z} \mathrm{~d} \lambda
$$

where it is assumed that the indicated densities exist, otherwise Stieltjes integrals involving distributions are required.

A key point is that the above eigenvalue characterization is implicit since the same term $m(z)$ appears on both the left and right hand sides of (8). As a consequence, while the work [2] is able to provide many insights into the nature of power control on DS-CDMA systems, it is only able to characterize the limiting SIR for the case of all the received powers being equal $\left(p_{k}=p\right)$ in which case the density $f(\lambda)$ becomes a Dirac delta $f(\lambda)=$ $\delta(\lambda-p)$ so that

$$
\int_{0}^{\infty} \frac{\tau f(\tau)}{1+\tau m(z)} \mathrm{d} \tau=\frac{p}{1+p m(z)}
$$

Noting that (6) and (9) imply $m\left(-\sigma^{2}\right)=\beta / p$, and substituting this and (10) into (8) evaluated at $z=-\sigma^{2}$ then indicates that in the equal power case

$$
\frac{\beta}{p}=\left(\frac{\alpha p}{1+\beta}+\sigma^{2}\right)^{-1}
$$

which is quadratic in $\beta$ with positive solution

$\beta=\frac{p}{2 \sigma^{2}}(1-\alpha)-\frac{1}{2}+\frac{1}{2 \sigma^{2}} \sqrt{p^{2}(1-\alpha)^{2}+2 p \sigma^{2}(1+\alpha)+\sigma^{4}}$

Unfortunately, it appears that this case of equal received powers, by virtue of the Dirac density it implies for $f(\lambda)$, is the only situation in which the characterization (8) may be applied in order to provide an explicit solution for the limiting $\operatorname{SIR} \beta$.

The purpose of this paper is to illustrate how an alternative strategy for analyzing (5), by means of avoiding direct characterization of the spectral distribution of the matrices involved, is able to provide an expression for $\beta$ which applies for arbitrary received power distributions. As an ancillary benefit, the arguments used also call upon less sophisticated mathematical ideas than the pre-existing approach just outlined.

\section{A NEW ANALYSIS}

The key tool which we apply to the analysis of (5) is the strong law of large numbers. Specifically, if $\left\{X_{k}\right\}$ is a sequence of independent random variables for which certain regularity conditions apply (see Theorem 2 in the Appendix), the strong law of large numbers asserts that

$$
\lim _{N \rightarrow \infty} \frac{1}{N} \sum_{k=1}^{N}\left(X_{k}-\mathbf{E}\left\{X_{k}\right\}\right)=0, \quad \text { w.p.1 }
$$

where the "with probability one" (w.p.1) epithet indicates that the above limit may only fail to hold on a subset $\Omega^{\prime} \subset \Omega$ (of the underlying probability space $\{\Omega, \mathcal{F}, \mathbf{P}\}$ on which the $\left\{X_{k}\right\}$ are defined) for which $P\left(\Omega^{\prime}\right)=0$. The intuitive understanding of this law is that if $E\left\{X_{k}\right\}=\bar{X}$ for all $k$, then for "large" $N$, the approximation

$$
\frac{1}{N} \sum_{k=1}^{N} X_{k} \approx \frac{1}{N} \sum_{k=1}^{N} \mathrm{E}\left\{X_{k}\right\}=\bar{X}
$$

is likely to be accurate.

The purpose of this section is to show how this principle can be used to provide insight into (5). While perhaps the first temptation would be to apply the strong law of large numbers to the 
$S D S^{T}$ term in (5) in an attempt to approximate it by a diagonal matrix, this approach is fraught with difficulty since, by construction, $S D S^{T}$ is rank deficient so that no diagonal expression is likely to be an accurate approximation. Indeed, one view of the existing work [2], [1] is that it deals with precisely this difficulty by way of eigenvalue distributions.

In recognition of these pitfalls, this paper takes an alternative approach by using the matrix inversion lemma [6], which states that

$$
[A+B C D]^{-1}=A^{-1}-A^{-1} B\left[C^{-1}+D A^{-1} B\right]^{-1} D A^{-1}
$$

for arbitrary matrices $A, B, C, D$ of compatible dimensions and such that indicated inverses exist. Application of this to (5) yields

$$
\beta_{N}=\frac{p_{1}}{\sigma^{2}} S_{1}^{T}\left[I-S\left(\sigma^{2} D^{-1}+S^{T} S\right)^{-1} S^{T}\right] S_{1} .
$$

Denoting by $[A]_{m, n}$ the $m, n$ 'th element of an arbitrary matrix $A$, then under the random variable model (7)

$$
\left[S^{T} S\right]_{m, n}=\frac{1}{N} \sum_{k=1}^{N} V_{m+1}(k) V_{n+1}(k) .
$$

Using $X_{k}=V_{m+1}(k) V_{n+1}(k)$ in the strong law of large numbers result (12) and, by the independence assumptions $\boldsymbol{E}\left\{X_{k}\right\}=\delta(m-n)$ with $\delta$ being the Kronecker delta, the following approximation (valid for large $N$ ) follows:

$$
S^{T} S \approx I \text {. }
$$

Thus for large $N$

$$
\beta_{N} \approx \frac{p_{1}}{\sigma^{2}}\left[S_{1}^{T} S_{1}-S_{1}^{T} S \Sigma S^{T} S_{1}\right]
$$

where

$$
\Sigma \triangleq \operatorname{diag}_{2 \leq \ell \leq K} \frac{1}{\sigma^{2} / p_{\ell}+1}
$$

Now, again by (7) and (12),

$$
S_{1}^{T} S_{1}=\frac{1}{N} \sum_{k=1}^{N} V_{1}^{2}(k) \approx \frac{1}{N} \sum_{k=1}^{N} \mathrm{E}\left\{V_{1}^{2}(k)\right\}=1 .
$$

Denoting

$$
\eta_{k} \triangleq \frac{1}{N} \sum_{n=1}^{N} V_{1}(n) V_{k}(n)
$$

it follows that

$$
S_{1}^{T} S \Sigma S^{T} S_{1}=\sum_{k=2}^{K} \frac{\eta_{k}^{2}}{\sigma^{2} / p_{k}+1}
$$

and therefore, again by (12) and now for large $K$,

$$
\frac{1}{K-1} S_{1}^{T} S \Sigma S^{T} S_{1} \approx \frac{1}{K-1} \sum_{k=2}^{K} \frac{\mathbf{E}\left\{\eta_{k}^{2}\right\}}{\sigma^{2} / p_{k}+1} .
$$

Furthermore, by the definition (18) and the independence and unit variance assumptions on $\left\{V_{k}(n)\right\}$,

$$
\begin{aligned}
\mathbf{E}\left\{\eta_{k}^{2}\right\} & =\frac{1}{N^{2}} \sum_{n=1}^{N} \sum_{m=1}^{N} \mathbf{E}\left\{V_{1}(n) V_{k}(n) V_{1}(m) V_{k}(m)\right\} \\
& =\frac{1}{N^{2}} \sum_{n=1}^{N} \mathbf{E}\left\{V_{1}^{2}(n) V_{k}^{2}(n)\right\}=\frac{1}{N}
\end{aligned}
$$

so that for large $K$ and $N$

$$
S_{1}^{T} S \Sigma S^{T} S_{1} \approx \frac{1}{N} \sum_{k=2}^{K} \frac{1}{\sigma^{2} / p_{k}+1} .
$$

Substituting (20) and (17) into (15) then provides the following large $N$ and $K$ approximation for SIR:

$$
\beta_{N} \approx \frac{p_{1}}{\sigma^{2}}\left[1-\frac{1}{N} \sum_{k=2}^{K} \frac{1}{\sigma^{2} / p_{k}+1}\right] .
$$

While this approximation holds for arbitrary distributions of the received powers $\left\{p_{k}\right\}$, it is useful to reconcile it with the existing approximation (11) that applies only for equal powers. Specifically, note that if $p_{k}=p$ for all $k$, then for SNR sufficiently high such that $\sigma^{2} / p \ll 1$, the approximation (21) becomes

$$
\beta_{N} \approx \frac{p_{1}}{\sigma^{2}}[1-\alpha]
$$

where $\alpha=K / N$. At the same time, under the same high SNR assumption, the square root in (11) is dominated by the first term so that it too implies (22) after recognising that the $-1 / 2$ term is negligible.

The somewhat heuristic arguments leading to (21) are now presented more formally in the following Theorem, which is the main result of the paper. The proof is given in the Appendix.

Theorem I: Let $\beta_{N}$ given by (5) be the random SIR of the MMSE receiver for user 1 . Then under the random spreading sequence model (7),

$$
\lim _{N, K \rightarrow \infty}\left|\beta_{N}-\frac{p_{1}}{\sigma^{2}}\left[1-\frac{1}{N} \sum_{k=2}^{K} \frac{1}{\sigma^{2} / p_{k}+1}\right]\right|=0
$$

with probability one.

The expression (21) then arises by assuming that for the $N$ and $K$ of interest, they are large enough that the convergence indicated in Theorem 1 has approximately occurred.

\section{SIMULATION RESULTS}

While Theorem 1 suggests the expression (21) for SIR, the accuracy of this expression will depend on whether the number of users $K$ and the spreading code length $N$ are both sufficiently large.

To test the validity of this approach for finite $N$ and $K$ that are likely to be encountered in practice, this paper presents a short simulation study in which the asymptotic limit (21) is compared with actual SIR's computed using equation (5) for randomly generated \pm 1 spreading sequences.

For each value of $\alpha$, the Monte Carlo simulation results are obtained by averaging the realised SIR's over 100 independently generated samples of the spreading sequence matrix 
$\left[S_{1}, S_{2}, \ldots, S_{K}\right]$. The spreading code length $N=128$, and the variance of the background Gaussian noise is $\sigma^{2}=0.01$.

Figure 1 displays results for the MMSE receiver assuming equal received powers from all users: $p_{i}=1$, so that the signalto-noise ratio $p / \sigma^{2}=20 \mathrm{~dB}$. Here the dash-dot line is the new expression (21), which is clearly an accurate approximation to the (Monte Carlo estimate of) the true SIR shown as the solid line. However, for large $\alpha$, note that it is not as accurate as the existing approximation (11), although the discrepancy is so small that (21) is still clearly informative.

If the received power distribution is non-constant, as is shown for example in the lower plot of Figure 2, then (11) is no longer valid, while (21) remains applicable and is shown as a dash-dot line. Clearly, it is an accurate approximation in this case as well.
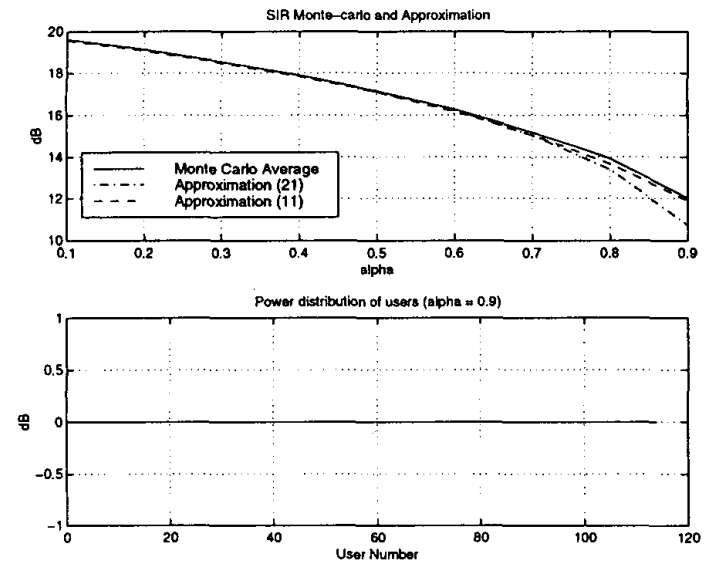

Fig. I. Upper plot: Monte Carlo average of randomly generated MMSE SIR's for user I as solid line, compared to approximate expressions (11) and (2l) shown (respectively) as dashed and dash-dot lines. Lower plot shows re ceived power distribution, which in this case is constant
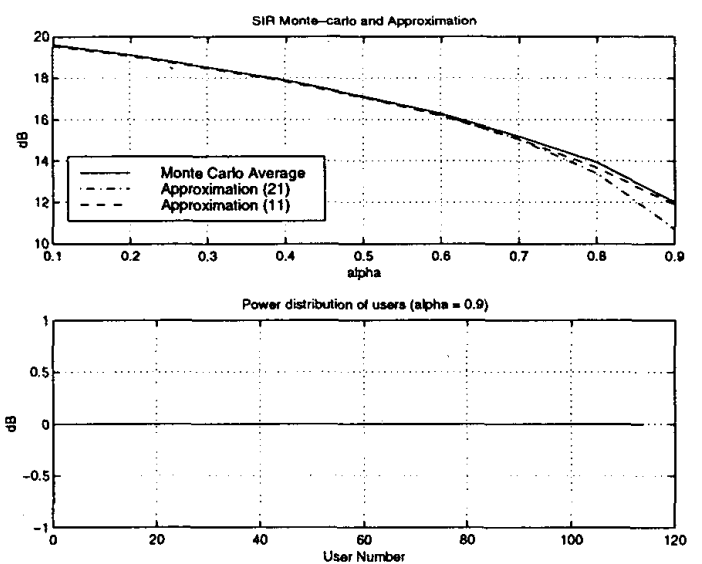

Fig. 2. Upper plot: Monte Carlo average of randomly generated MMSE SIR's for user I as solid line, compared to appmoximate expressions (1I) and (2I) shown (respectively) as dashed and dash-dot lines. Lower plot shows received power distribution.

\section{APPENDIX}

\section{TECHNiCAL RESUlts}

Theorem 2 (A Strong Law of Large Numbers) Suppose $\left\{X_{t}\right\}$ is a sequence of random variables, not necessarily zero mean, and with arbitrary correlation structure (not necessarily stationary) that is characterized by the existence of a $C<\infty$, $1<\beta<\infty$ such that

$$
\sum_{t=1}^{N} \sum_{s=1}^{N} \mathrm{E}\left\{X_{t} X_{s}\right\} \leq C N^{\beta} .
$$

Then for any $\alpha>\beta / 2$

$$
\frac{1}{N^{\alpha}} \sum_{t=1}^{N} X_{t} \stackrel{\text { a.s. }}{\longrightarrow} 0 \quad \text { as } N \rightarrow \infty .
$$

Proof: See [7] for a proof of the Theorem as stated, or see Theorem 3.7.2 [8] for a slightly weaker result that is still adequate for the purposes of this paper.

\section{Proof of Main Theorem}

Proof: In what follows, $C$ will denote an unspecified but guaranteed finite quantity that may be different in different parts of the proof. Returning to the formulation (13)

$$
\beta_{N}=\frac{p_{1}}{\sigma^{2}} S_{1}^{T}\left[I-S\left(\sigma^{2} D^{-1}+S^{T} S\right)^{-1} S^{T}\right] S_{1},
$$

then initially focusing on (14), which states that

$$
\left[S^{T} S\right]_{m, n}=\frac{1}{N} \sum_{k=1}^{N} V_{m+1}(k) V_{n+1}(k)
$$

use of the assumptions on $\left\{V_{n}(k)\right\}$ yields

$$
\mathbf{E}\left\{V_{m+1}(k) V_{n+1}(k)\right\}=\delta(m-n)
$$

for $\delta$ equal to the Kronecker delta, and

$$
\begin{aligned}
& \mathbf{E}\left\{V_{m+1}(k) V_{n+1}(k) V_{m+1}(j) V_{n+1}(j)\right\}= \\
& \begin{cases}\delta(k-j) & ; m \neq n \\
1 \quad ; k \neq j & ; m=n \\
\kappa ; k=j & \end{cases}
\end{aligned}
$$

so that

$$
\mathbf{E}\left\{\left|\sum_{k=1}^{N} V_{m+1}(k) V_{n+1}(k)-N \delta(m-n)\right|^{2}\right\} \leq C N .
$$

Therefore, by Theorem 2 and for some $\delta>0$

$$
\lim _{N \rightarrow \infty} \frac{1}{N^{1 / 2+\delta}}\left|\sum_{k=1}^{N} V_{m+1}(k) V_{n+1}(k)-N \delta(m-n)\right|=0, \quad \text { w.p.1 }
$$

so that by (B.24)

$$
\left|\left[S^{T} S\right]_{m, n}-\delta(m-n)\right| \leq \frac{C}{N^{1 / 2}-\delta}, \quad \text { w.p. } 1 .
$$

For an arbitrary square matrix $A \in \mathbf{R}^{n \times n}$, define the norm $\|A\|$ to be the spectral norm $\|A\|=\sup _{x \in \mathbf{R}^{n}} x^{T} A x / x^{T} x$. Then 
combining the above result with $[9$, equation (2.3.8)] and with probability one

$$
S^{T} S=I+\Delta_{1}, \quad\left\|\Delta_{1}\right\| \leq C \frac{K}{N^{1 / 2-\delta}} .
$$

Using an identical argument together with the formulation (17) and Theorem 2

$$
S_{1}^{T} S_{1}=1+\Delta_{2}, \quad\left|\Delta_{2}\right| \leq \frac{C}{N^{1 / 2-\delta}}, \quad \text { w.p.1. }
$$

Substituting these expressions into (13) and using the Matrix Inversion Lemma then provides

$$
\begin{aligned}
\beta_{N}= & \frac{p_{1}}{\sigma^{2}}\left\{1-S_{1}^{T} S \Sigma S^{T} S_{1}-\right. \\
& \left.S_{1}^{T} S\left(\Sigma+\Delta_{1}\right)^{-1} \Delta_{1} \Sigma S^{T} S_{1}\right\}+\Delta_{2} \frac{p_{1}}{\sigma^{2}} \text { (B.26) }
\end{aligned}
$$

where $\Sigma$ is given by (16). Now, with the definition (18) of $\eta_{k}$, it follows from (19) that

$$
S_{1}^{T} S \Sigma S^{T} S_{1}=\sum_{k=2}^{K} \frac{\eta_{k}^{2}}{\sigma^{2} / p_{k}+1}
$$

and under the independence, unit variance, and finite fourth moment assumptions on $\left\{V_{k}(n)\right\}$

$$
\begin{aligned}
\mathbf{E}\left\{\eta_{k}^{2}\right\} & =\frac{1}{N^{2}} \sum_{n=1}^{N} \sum_{m=1}^{N} \mathbf{E}\left\{V_{1}(n) V_{1}(m) V_{k}(n) V_{k}(m)\right\} \\
& =\frac{1}{N^{2}} \sum_{n=1}^{N} \mathbf{E}\left\{V_{1}(n)^{2}\right\} \mathbf{E}\left\{V_{k}(n)^{2}\right\}=\frac{1}{N}(\mathrm{~B} .27)
\end{aligned}
$$

Furthermore,

$$
\begin{aligned}
\mathbf{E}\left\{\eta_{k}^{2} \eta_{j}^{2}\right\}= & \frac{1}{N^{4}} \sum_{n, m, \ell, r=1}^{N} \mathbf{E}\left\{V_{1}(n) V_{1}(m) V_{1}(\ell) V_{1}(r)\right\} \times \\
& \mathbf{E}\left\{V_{k}(m) V_{k}(n) V_{j}(\ell) V_{j}(r)\right\}
\end{aligned}
$$

so that when $k \neq j$

$$
\begin{aligned}
\mathbf{E}\left\{\eta_{k}^{2} \eta_{j}^{2}\right\} & =\frac{1}{N^{4}} \sum_{n=1}^{N} \sum_{\ell=1}^{N} \mathbf{E}\left\{V_{1}^{2}(n) V_{1}^{2}(\ell)\right\} \\
& =\frac{1}{N^{4}}(N \kappa+N(N-1))
\end{aligned}
$$

while when $k=j$

$$
\mathbf{E}\left\{\eta_{k}^{2} \eta_{j}^{2}\right\}=\frac{1}{N^{4}}\left[N \kappa^{2}+N(3 N-1)\right]
$$

where the last line follows since the expectations will be zero unless there are two matched pairs of indices, and taking first index $n$, as it ranges through $N$ values, it can match 3 other indices, each of which can range through $N$ possible values. Therefore, there are $3 N^{2}$ times the quadruple summation involves pairs of matched indices, and of these, $N$ occurrences involve all four indices being matched. Therefore

$$
\mathbf{E}\left\{\eta_{k}^{2} \eta_{j}^{2}\right\}=\left\{\begin{array}{cc}
\frac{1}{N^{3}}\left[\kappa^{2}+(3 N-1)\right] & ; k=j \\
\frac{1}{N^{3}}[\kappa+(N-1)] & ; k \neq j
\end{array}\right.
$$

and hence for some $C<\infty$

$$
\sum_{k=2}^{K} \sum_{j=2}^{K} \frac{\mathbf{E}\left\{\left(N \eta_{k}^{2}-1\right)\left(N \eta_{j}^{2}-1\right)\right\}}{\left(\sigma^{2} / p_{k}+1\right)\left(\sigma^{2} / p_{j}+1\right)} \leq C K^{2} .
$$

Therefore, by Theorem 2, and for some $\delta>0$

$$
\frac{1}{K^{1+\delta}} \sum_{k=2}^{K} \frac{N \eta_{k}^{2}-1}{\sigma^{2} / p_{k}+1}=0, \quad \text { w.p. } 1
$$

so that since

$$
N\left(S_{1}^{T} S \Sigma S^{T} S_{1}-\frac{1}{N} \sum_{k=2}^{K} \frac{1}{\sigma^{2} / p_{k}+1}\right)=\sum_{k=2}^{K} \frac{N \eta_{k}^{2}-1}{\sigma^{2} / p_{k}+1}
$$

then for some $C<\infty$ and with probability one

$S_{1}^{T} S \Sigma S^{T} S_{1}=\frac{1}{N} \sum_{k=2}^{K} \frac{1}{\sigma^{2} / p_{k}+1}+\Delta_{3}, \quad\left|\Delta_{3}\right| \leq C \frac{K^{1+\delta}}{N}$.

Therefore, using (B.26)

$$
\begin{gathered}
\left|\beta^{N}-\frac{p_{1}}{\sigma^{2}}\left(1-\frac{1}{N} \sum_{k=2}^{K} \frac{1}{\sigma^{2} / p_{k}+1}\right)\right|= \\
\left|\frac{p_{1}}{\sigma^{2}}\left[\Delta_{3}+\Delta_{2}-S_{1}^{T} S\left(\Sigma+\Delta_{1}\right)^{-1} \Delta_{1} \Sigma S^{T} S_{1}\right]\right| .
\end{gathered}
$$

Finally, by the definition of the matrix norm (induced 2-norm) and the Cauchy-Schwarz inequality,

$\left|S_{1}^{T} S\left(\Sigma+\Delta_{1}\right)^{-1} \Delta_{1} \Sigma S^{T} S_{1}\right| \leq\left\|\Delta_{1}\right\| \cdot\left\|\left(\Sigma+\Delta_{1}\right)^{-1}\right\| \cdot\|\Sigma\| S_{1}^{T} S S^{T} S_{1}$.

However, as just established

$$
\left|S_{1}^{T} S S^{T} S_{1}\right|=\left|\sum_{k=2}^{K} \eta_{k}^{2}\right| \leq \Delta_{3}, \quad \text { w.p.1 }
$$

so that assuming the power distribution is such that $\Sigma>0$ and noticing that matrix inversion is continuous with respect to the matrix 2-norm then completes the proof.

\section{REFERENCES}

(1) S. Verdú and S. Shamai (Schitz), "Spectral efficiency of cdma with random spreading," IEEE Transactions on Information Theory, vol. 45, pp. 622640, March 1999.

[2] D. N. C. Tse and S. V. Hanly, "Linear multiuser receivers: Effective interference, effective bandwidth and user capacity," IEEE Transactions on Information Theory, vol. 45, pp. 641-657, March 1999.

[3] P. Viswanath, V. Anantharam, and D. N. C. Tse, "Optimal sequences, power control, and user capacity of synchronous CDMA systems with linear MMSE multiuser receivers," IEEE Trans. Inform. Theory, vol. 45, no. 6 , pp. 1968-1983, 1999.

[4] J. W. Silverstein, "On the empirical distribution of eigenvalues of a class of large dimensional random matrices," Journal of Multivariate Analysis, vol. 54, pp. 175-192, 1995.

[5] U. Madhow and M. L. Honig, "Mmse interference suppresion for directsequence spread-spectrum cdma," IEEE Transactions on Communications, vol. 42, no. 12, pp. 3178-3188, 1994.

[6] A. Weinmann, Uncertain Models and Robust Control. New York: SpringerVerlag, 1991.

[7] B. Ninness, "Strong laws of large numbers under weak assumptions with applications," To appear, IEEE Transactions on Automatic Control. Preprint applications, To appear, IEEE Transactions on Automatic Cont
available at www.ee.newcastle.edu.aw/users/staff/brett, 2000.

[8] W. F. Stout, Almost Sure Convergence. Academic Press, 1974

[9] G. Golub and C. V. Loan, Matrix Computations. Johns Hopkins University Press, 1989. 